\title{
Proliferative diabetic retinopathy is associated with microalbuminuria in patients with type 2 diabetes
}

M.C. Boelter ${ }^{2}$, J.L. Gross ${ }^{1}$, L.H. Canani ${ }^{1}$, L.A. Costa ${ }^{1}$, H.R. Lisboa ${ }^{3}$, G.S. Três ${ }^{3}$, J. Lavinsky ${ }^{2}$ and M.J. Azevedo ${ }^{1}$
${ }^{1}$ Serviço de Endocrinologia, ${ }^{2}$ Serviço de Oftalmologia, Hospital de Clínicas de Porto Alegre, Universidade Federal do Rio Grande do Sul, Porto Alegre, RS, Brasil

${ }^{3}$ Departamento de Clínica Médica, Universidade de Passo Fundo, Passo Fundo, RS, Brasil

\section{Correspondence \\ M.J. Azevedo \\ Serviço de Endocrinologia \\ Hospital de Clínicas de Porto Alegre \\ Rua Ramiro Barcelos, 2350 \\ Prédio 12, 4으andar \\ 90035-003 Porto Alegre, RS \\ Brasil \\ Fax: +55-51-3332-5188/3330-9100 \\ E-mail: mirelaazevedo@terra.com.br \\ Research supported by CNPq \\ (Programa de Apoio a Núcleos de \\ Excelência, No. 6610621996/1) \\ and Hospital de Clínicas de Porto \\ Alegre (FIPE-HCPA, No. 0407-5). \\ L.A. Costa was the recipient of a \\ scholarship from CAPES and \\ L.H. Canani was supported by a \\ grant from CAPES (PRODOC, No. \\ 127/03-5).}

Received November 3, 2005

Accepted May 29, 2006

\begin{abstract}
Diabetic retinopathy is one of the leading causes of blindness in working-age individuals. Diabetic patients with proteinuria or those on dialysis usually present severe forms of diabetic retinopathy, but the association of diabetic retinopathy with early stages of diabetic nephropathy has not been entirely established. A cross-sectional study was conducted on 1214 type 2 diabetic patients to determine whether microalbuminuria is associated with proliferative diabetic retinopathy in these patients. Patients were evaluated by direct and indirect ophthalmoscopy and grouped according to the presence or absence of proliferative diabetic retinopathy. The agreement of diabetic retinopathy classification performed by ophthalmoscopy and by stereoscopic color fundus photographs was 95.1\% (kappa $=0.735 ; \mathrm{P}<0.001$ ). Demographic information, smoking history, anthropometric and blood pressure measurements, glycemic and lipid profile, and urinary albumin were evaluated. On multiple regression analysis, diabetic nephropathy $(\mathrm{OR}=5.18,95 \% \mathrm{CI}=2.91-9.22, \mathrm{P}<0.001)$, insulin use $(\mathrm{OR}=2.52,95 \% \mathrm{CI}=1.47-4.31, \mathrm{P}=0.001)$ and diabetes duration $(\mathrm{OR}$ $=1.04,95 \% \mathrm{CI}=1.01-1.07, \mathrm{P}=0.011)$ were positively associated with proliferative diabetic retinopathy, and body mass index ( $\mathrm{OR}=$ $0.90,95 \% \mathrm{CI}=0.86-0.96, \mathrm{P}<0.001$ ) was negatively associated with it. When patients with macroalbuminuria and on dialysis were excluded, microalbuminuria $(\mathrm{OR}=3.3,95 \% \mathrm{CI}=1.56-6.98, \mathrm{P}=0.002)$ remained associated with proliferative diabetic retinopathy. Therefore, type 2 diabetic patients with proliferative diabetic retinopathy more often presented renal involvement, including urinary albumin excretion within the microalbuminuria range. Therefore, all patients with proliferative diabetic retinopathy should undergo an evaluation of renal function including urinary albumin measurements.
\end{abstract}

Key words

- Diabetic retinopathy

- Diabetic nephropathy

- Microalbuminuria

- Type 2 diabetes mellitus 


\section{Introduction}

Diabetic retinopathy (DR) is the leading cause of new cases of legal blindness among working-age individuals in the United States. The more advanced the DR, the greater the risk of visual loss. Furthermore, patients with DR are at higher risk for coronary heart disease, stroke, diabetic nephropathy, limb amputations, and death (1). The main risk factors for the development or progression of DR are duration of diabetes mellitus (2), poor glycemic control $(2,3)$ and hypertension $(4,5)$.

Increasing evidence suggests that DR has a genetic component. However, so far most candidate genes studied have exhibited a weak or no association with DR, and the positive associations detected have not been confirmed in subsequent studies (6).

Diabetic patients with proteinuria or those on dialysis usually present severe forms of DR $(2,7,8)$, but the association of DR with early stages of diabetic nephropathy has not been entirely established. Although microalbuminuria has been associated with an increased risk of proliferative DR in type 1 diabetic patients (9), this association is the subject of controversy for type 2 diabetic patients $(5,8,10-13)$.

The aim of the present study was to determine if microalbuminuria is associated with proliferative DR in type 2 diabetic patients while taking into account other possible factors.

\section{Material and Methods}

\section{Ophthalmologic evaluation}

A cross-sectional study was conducted on 1214 type 2 diabetic outpatients defined according to WHO criteria. The patients were attended from 2002 to 2004 at the Endocrinology Clinics of two general university hospitals, Hospital de Clínicas de Porto Alegre $(\mathrm{N}=858)$ and Hospital São
Vicente de Paula $(\mathrm{N}=356)$ in the State of Rio Grande do Sul, Brazil. Informed written consent was obtained from all patients. The Ethics Committee at Hospital de Clínicas de Porto Alegre approved the study protocol.

Fundus examination was performed in all patients by a trained ophthalmologist using direct and indirect ophthalmoscopy through dilated pupils. Retinopathy was classified as non-proliferative (including absent signs of DR or signs of non-proliferative $\mathrm{DR}$, i.e., microaneurysms, hemorrhage, hard exudates) or proliferative (newly formed blood vessels and/or growth of fibrous tissue into the vitreous cavity). Patients with panphotocoagulation were classified as presenting proliferative DR. The severity of DR was graded based on the worst eye. In 2 patients in whom the presence of media opacities due to vitreous hemorrhage (1 patient), and cataract (1 patient) prevented fundoscopy in one eye, the contralateral eye was used to classify DR. No patient was excluded as a result of unreadable fundoscopy in both eyes. The diagnosis of proliferative DR based on fundoscopy performed by the ophthalmologists was used to classify the patients.

A subset of 240 patients from Hospital de Clínicas de Porto Alegre, selected for reasons of convenience, underwent stereoscopic color fundus photographs of seven standard fields (14) to analyze the agreement between the classification of retinopathy using this method and ophthalmoscopy performed by the physicians. Initially, the ophthalmologists (MCB and JLG), who were unaware of the patients' clinical data, classified the fundus photographs independently according to the criteria of the American Academy of Ophthalmology (AAO) (15). The agreement of DR classification performed by ophthalmoscopy and stereoscopic fundus photographs was then analyzed to validate the ophthalmoscopy procedure used to classify studied patients. 


\section{Clinical and laboratory evaluation}

Age, sex, ethnicity (self-definition as white or non-white), known duration of diabetes, diabetes treatment, and smoking habits were evaluated. Patients were considered to be smokers if they were current smokers. Body mass index (BMI; weight $(\mathrm{kg}) /$ height $^{2}$ (m)) and waist-to-hip ratio (WHR) were calculated. Hypertension was considered to be present when blood pressure $\geq 140 / 90 \mathrm{mmHg}$ or when antihypertensive drugs were in use. The diagnosis of metabolic syndrome (WHO criteria) was made in the presence of 2 or more of the following parameters: blood pressure $\geq 140 / 90 \mathrm{mmHg}$, plasma triglycerides $\geq 150 \mathrm{mg} / \mathrm{dL}$ and/or HDL cholesterol $<35 \mathrm{mg} / \mathrm{dL}$ for men or $<39 \mathrm{mg} / \mathrm{dL}$ for women, BMI $>30 \mathrm{~kg} / \mathrm{m}^{2}$ and/or WHR $>0.9$ for males and $>0.85$ for females, and microalbuminuria.

$\mathrm{HbA}_{\mathrm{lc}}$ (HPLC, reference range: 4.7-6.0\%; Merck-Hitachi L-9100 glycated hemoglobin analyzer, Merck, Darmstadt, Germany), creatinine (Jaffe's reaction, autoanalyzer ADVIA1650, Bayer Diagnostic, Tarrytown, NY, USA), triglycerides and total cholesterol (enzymatic colorimetric method, Merck Diagnostica, Darmstadt, Germany; Boehringer Mannheim, Buenos Aires, Argentina), HDL (homogeneous direct method, autoanalyzer ADVIA1650) and LDL (Friedewald equation) were measured.

Albuminuria was measured by immunoturbidimetry (MicroAlb SeraPak ${ }^{\circledR}$ Bayer, Tarrytown, NY, USA on Cobas Mira Plus $\left(\right.$ Roche $\left.^{\circledR}\right)$. Urinary albumin was measured in a sterile random urine sample $(40.2 \%$ of samples) or in a 24 -h sample, timed $(45.0 \%$ of samples) or untimed (14.8\% of samples). Diabetic nephropathy was diagnosed in the presence of microalbuminuria (albumin $=$ $17-176 \mathrm{mg} / \mathrm{L}$ in a random urine sample, or 30-299 mg in a 24-h urine collection, or 24h urinary albumin excretion rate (UAER) $=$ 20 to $199 \mu \mathrm{g} / \mathrm{min}$ ) or macroalbuminuria (albumin $\geq 176 \mathrm{mg} / \mathrm{L}$ in a random urine sample, or $\geq 300 \mathrm{~g}$ in a 24 -h urine collection or 24-h UAER $\geq 200 \mu \mathrm{g} / \mathrm{min}$ ) (16), or in patients on dialysis. Albuminuria values in the range of micro- or macroalbuminuria were confirmed in a second urine specimen.

\section{Statistical analysis}

The unpaired Student $t$-test, the MannWhitney U-test, the chi-square $\left(\chi^{2}\right)$ test, and one-way analysis of variance (ANOVA) were used when appropriate. The kappa coefficient was used to assess the agreement between DR classification by different ophthalmologists and by different methods (stereoscopic fundus photographs and ophthalmoscopy), and to evaluate the agreement of DR classification performed by the same ophthalmologist on two separate occasions in a subset of 110 patients. Multiple logistic regression models were used to evaluate factors associated with proliferative DR. Results are reported as means $\pm \mathrm{SD}$, as percentage of patients with the characteristic or as median (range). P values $<0.05$ were considered to be significant. The SPSS statistical package 10.0 and Epi-Info, version 6.04d, were used for the analysis.

\section{Results}

\section{Clinical and laboratory characteristics of the 1214 patients}

Most patients with proliferative DR were male, older, presenting longer duration of diabetes, less frequently were smokers, and had a higher prevalence of metabolic syndrome than patients without proliferative DR (Table 1). Patients with proliferative DR more frequently used insulin (alone or in combination with oral agents) and angiotensin-converting enzyme inhibitors, and had hypertension and diabetic nephropathy (macroalbuminuria and dialysis) than patients without proliferative DR. These patients also had higher systolic blood pressure levels, 
lower BMI and HDL values, and higher serum creatinine levels than patients without proliferative DR. However, the two groups were similar regarding diastolic blood pressure levels, WHR, $\mathrm{HbA}_{1 \mathrm{c}}$, total cholesterol, LDL, and triglycerides.

Proliferative DR was diagnosed in 54 of 240 patients in the subset submitted to fundus photographs; the remaining 186 patients did not present proliferative DR (no retinopathy in 125 patients, mild non-proliferative DR in 39 patients, moderate non-proliferative DR in 9 patients, severe and severe non-proliferative DR in 13 patients). Thus, the proportion of patients with proliferative DR was similar in patients with and without fundus photographs (22.5 vs 18.7\%; $\mathrm{P}=$ $0.204)$.

Table 1. Clinical and laboratory characteristics of 1214 type 2 diabetic patients with and without proliferative diabetic retinopathy.

\begin{tabular}{lccr}
\hline & $\begin{array}{c}\text { With proliferative } \\
\text { diabetic retinopathy }\end{array}$ & $\begin{array}{c}\text { Without proliferative } \\
\text { diabetic retinopathy }\end{array}$ & $\mathrm{P}$ \\
& & & \\
Number of subjects & 227 & 987 & \\
Male sex & $134(59.0 \%)$ & $392(39.7 \%)$ & $<0.001^{\mathrm{a}}$ \\
Age (years) & $60.3 \pm 9.9$ & $58.1 \pm 10.4$ & $0.005^{\mathrm{b}}$ \\
Diabetes duration (years) & $15.6 \pm 8.5$ & $10.1 \pm 8.1$ & $<0.001^{\mathrm{b}}$ \\
Smoking habit & $30(13.2 \%)$ & $203(20.6 \%)$ & $0.016^{\mathrm{a}}$ \\
Metabolic syndrome & $209(92.2 \%)$ & $806(81.7 \%)$ & $<0.001^{\mathrm{a}}$ \\
Insulin use & $143(63.0 \%)$ & $319(32.3 \%)$ & $<0.001^{\mathrm{a}}$ \\
Use of ACE inhibitors & $121(53.1 \%)$ & $366(37.1 \%)$ & $<0.001^{\mathrm{a}}$ \\
Hypertension & $179(78.7 \%)$ & $649(65.8 \%)$ & $<0.001^{\mathrm{a}}$ \\
Diabetic nephropathy & $180(79.3 \%)$ & $347(35.2 \%)$ & $<0.001^{\mathrm{a}}$ \\
Microalbuminuria & $47(20.7 \%)$ & $232(23.5 \%)$ & $0.416^{\mathrm{a}}$ \\
Macroalbuminuria & $61(26.8 \%)$ & $96(9.7 \%)$ & $<0.001^{\mathrm{a}}$ \\
Dialysis & $72(31.7 \%)$ & $19(1.9 \%)$ & $<0.001^{\mathrm{a}}$ \\
Systolic blood pressure (mmHg) & $147.2 \pm 22.7$ & $141.4 \pm 23.9$ & $0.002^{\mathrm{b}}$ \\
Diastolic blood pressure (mmHg) & $85.6 \pm 14.3$ & $86.7 \pm 13.5$ & $0.307^{\mathrm{b}}$ \\
Body mass index (kg/m $\left.{ }^{2}\right)$ & $27.4 \pm 4.7$ & $29.4 \pm 5.4$ & $<0.001^{\mathrm{b}}$ \\
Waist-to-hip ratio & $0.95 \pm 0.08$ & $0.95 \pm 0.07$ & $0.362^{\mathrm{b}}$ \\
Glycosylated hemoglobin $(\%)$ & $8.62 \pm 1.84$ & $8.12 \pm 2.03$ & $0.864^{\mathrm{b}}$ \\
Total cholesterol (mg/dL) & $212.7 \pm 54.1$ & $212.7 \pm 54.0$ & $0.864^{\mathrm{b}}$ \\
HDL (mg/dL) & $42.5 \pm 11.6$ & $46.4 \pm 11.0$ & $0.042^{\mathrm{b}}$ \\
LDL (mg/dL) & $143.1 \pm 54.1$ & $139.2 \pm 38.7$ & $0.208^{\mathrm{b}}$ \\
Triglycerides (mg/dL) & $150.6(53.1-903.4)$ & $150.7(26.6-1470.0)$ & $0.977^{\mathrm{c}}$ \\
Serum creatinine (mg/dL) & $1.48 \pm 1.14$ & $0.97 \pm 0.32$ & $<0.001^{\mathrm{b}}$ \\
& & &
\end{tabular}

Data are reported as mean $\pm \mathrm{SD}$, median (range) or number (percentage) of patients with the characteristic. ACE = angiotensin-converting enzyme; diabetic nephropathy = micro- and macroalbuminuric patients, and patients on dialysis. Patients on dialysis were excluded from the analysis of serum creatinine.

${ }^{a} \chi^{2}$ test; bunpaired $t$-test; cMann-Whitney U-test.
The agreement of DR classification carried out by stereoscopic fundus photographs performed by the different ophthalmologists (MCB and JLG) was 95.3\% (kappa = 0.774; $\mathrm{P}<0.001)$ when using the simplified DR classification (presence or absence of proliferative DR), and $88.8 \%$ (kappa $=0.771 ; \mathrm{P}<$ 0.001 ) when using the AAO classification (15). Moreover, the agreement of DR classification performed by ophthalmoscopy and by stereoscopic fundus photographs was 95.1\% (kappa $=0.735 ; \mathrm{P}<0.001$ ) for the simplified classification, and $84.3 \%$ (kappa $=0.698 ; \mathrm{P}<0.001)$ for AAO classification (15).

\section{Multivariate analysis}

Multiple logistic regression models were used to analyze the possible associated factors for proliferative DR (Table 2). The presence of diabetic nephropathy (micro- or macroalbuminuria and dialysis), insulin use and known duration of diabetes were positively associated, while BMI was negatively associated with proliferative DR. Smoking habit had a borderline negative association with proliferative DR. Male sex, use of ACE inhibitors, HDL levels and systolic blood pressure were not associated with proliferative DR. The inclusion of glycosylated hemoglobin in the model did not change these results. To evaluate the association of microalbuminuria with proliferative DR, patients with macroalbuminuria $(\mathrm{N}=157)$ and patients on dialysis $(\mathrm{N}=91)$ were excluded from the regression model (Table 2). Microalbuminuria, insulin use and BMI were the variables associated with the presence of proliferative DR.

\section{Discussion}

In this sample of type 2 diabetic patients, proliferative DR was associated with increased levels of albuminuria, insulin use and lower BMI, even when duration of dia- 
betes, blood pressure levels and degree of glycemic control were taken into account. These associations were already evident when the UAER was still within the microalbuminuria range.

Despite the well-known association between advanced DR stages and macroalbuminuria (or proteinuria) in type 2 diabetic patients $(2,7,8,10)$, the relationship with lower levels of UAE within the range of microalbuminuria is controversial. Some investigators have reported a positive association $(8,10,12)$, while in other studies this has not been observed $(5,7,11,13)$. Some of these negative studies included highly selected patients who were not taking anti-hypertensive drugs, did not smoke and were younger (5) or presented shorter duration of diabetes $(7,13)$ and worse glycemic control (7) than the patients in the present study. If these aspects are taken into account, our patients could probably be considered to be representative of type 2 diabetic patients with proliferative DR. Furthermore, in some of these negative studies, patients with different degrees of DR were analyzed together $(5,13)$. The association between proliferative DR and microalbuminuria observed in the present study could be explained by the view that microalbuminuria might represent a state of generalized vascular dysfunction (17). Alternatively, microalbuminuria and DR may share common determinants. In fact, duration of diabetes and blood pressure levels are well-known risk factors for both DR and diabetic nephropathy $(3,4)$.

Recent data indicate that glycosylated hemoglobin levels are determinant for incident retinopathy in diabetic and non-diabetic populations (18). In fact, glycemic control was a risk factor for proliferative DR in the UKPDS prospective observational study (19), but the proportion of patients with diabetic nephropathy at baseline (approximately $14 \%$ ) was lower than that observed in the present study (43.8\%). The association of glycemic control with proliferative
DR was less evident in the present study probably because of the remarkable effect of the presence of renal disease itself.

Interestingly, the use of insulin was associated with the presence of DR. Other studies have also reported this association $(5,10)$. Type 2 diabetic patients using insulin in whom it is more difficult to achieve metabolic control usually present a significant decline in beta cell function (20). Poor glycemic control with oral agents is usually the main reason to start insulin treatment, and it is very unlikely that insulin per se could be implicated in the development of DR. In fact, in the UKPDS study (21), the intensive glycemic control obtained with insulin was associated with lower development of DR. Therefore, poorer glycemic control prior to insulin use was probably the main reason for the association between DR and insulin treatment observed in this sample of type 2 diabetic patients.

\begin{tabular}{|c|c|c|c|}
\hline Independent variables & OR & $95 \% \mathrm{Cl}$ & $\mathrm{P}$ \\
\hline \multicolumn{4}{|c|}{ Normo-, micro-, and macroalbuminuric patients and patients on dialysis $(\mathrm{N}=1214)$} \\
\hline Diabetic nephropathy & 5.18 & $2.91-9.22$ & $<0.001$ \\
\hline Insulin use & 2.52 & $1.47-4.31$ & 0.001 \\
\hline Body mass index $\left(\mathrm{kg} / \mathrm{m}^{2}\right)$ & 0.90 & $0.86-0.96$ & $<0.010$ \\
\hline Diabetes duration (years) & 1.04 & $1.01-1.07$ & 0.011 \\
\hline Male sex & 1.31 & $0.74-2.32$ & 0.361 \\
\hline Smoking habit & 0.44 & $0.20-1.00$ & 0.050 \\
\hline Systolic blood pressure $(\mathrm{mmHg})$ & 1.00 & $0.99-1.02$ & 0.855 \\
\hline Use of ACE inhibitors & 1.19 & $0.71-2.00$ & 0.521 \\
\hline $\mathrm{HDL}(\mathrm{mg} / \mathrm{dL})$ & 1.00 & $0.98-1.02$ & 0.770 \\
\hline \multicolumn{4}{|c|}{ Normo- and microalbuminuric patients ( $\mathrm{N}=966)$} \\
\hline Microalbuminuria & 3.30 & $1.56-6.98$ & 0.002 \\
\hline Insulin use & 2.25 & $1.05-4.81$ & 0.037 \\
\hline Body mass index $\left(\mathrm{kg} / \mathrm{m}^{2}\right)$ & 0.82 & $0.75-0.91$ & $<0.001$ \\
\hline Diabetes duration (years) & 1.04 & $1.00-1.09$ & 0.055 \\
\hline Male sex & 0.54 & $0.22-1.29$ & 0.163 \\
\hline Smoking habit & 0.56 & $0.12-1.75$ & 0.320 \\
\hline Systolic blood pressure $(\mathrm{mmHg})$ & 1.01 & $1.00-1.03$ & 0.186 \\
\hline Use of ACE inhibitors & 1.47 & $0.69-3.12$ & 0.319 \\
\hline $\mathrm{HDL}(\mathrm{mg} / \mathrm{dL})$ & 0.99 & $0.96-1.02$ & 0.462 \\
\hline
\end{tabular}

Dependent variable: presence of proliferative diabetic retinopathy. $\mathrm{OR}=$ odds ratio; $95 \% \mathrm{Cl}=$ confidence interval at 95\%; ACE = angiotensin-converting enzyme. $\mathrm{P}$ values refer to statistical significance of the association of each independent variable with the presence of proliferative diabetic retinopathy. 
An unexpected observation was the protective effect of BMI against proliferative DR, since metabolic syndrome, of which obesity is a major component, was positively associated with DR in the present study. In fact, we had already described this association (22). However, when patients with normal and abnormal glucose metabolism were followed for 9.4 years, BMI was found not to be associated with the development of any degree of retinopathy (18). Also, in a population-based study, BMI was not related to progression of DR and, in contrast, being underweight was associated with a higher incidence of DR (23). In that study only patients diagnosed as having diabetes when they were 30 years or older were evaluated. However, about $46 \%$ of them were taking insulin, and probably type 1 diabetic patients were also included. Therefore, the role of BMI as a risk factor for DR, especially advanced DR forms, is still undefined.

A borderline negative association of current smoking with proliferative DR suggests a potential protective role of smoking on DR, as also reported by others (3). Nevertheless, the specific reasons for this effect are still unclear.

Although systolic blood pressure levels were higher in the patients with proliferative DR in univariate analyses, there was no association of blood pressure with prolifera- tive DR. This observation is in consonance with UKPDS results (3), which did not reveal any relation between the progression of retinopathy and blood pressure levels in patients who already presented DR at baseline.

A possible limitation of the present study was the inclusion of individuals from tertiary care centers, favoring the selection of patients with more severe forms of diabetes and its complications. Also, it should be taken into account that microalbuminuria is not only associated with diabetic nephropathy but could also be related to other clinical conditions such as hypertension. However, the characteristics and proportion of microalbuminuric patients in the reference group (patients without or with non-severe forms of DR) were similar to those of populationbased studies. Furthermore, the inclusion of patients with a higher prevalence of complications, such as renal disease or even hypertension, in the group of patients without proliferative DR, would decrease the chance of a positive association between proliferative DR and microalbuminuria.

Type 2 diabetic patients with proliferative DR more often presented renal involvement, including increased UAER within the microalbuminuria range. Therefore, all patients with proliferative DR should undergo an evaluation of renal function that comprises urinary albumin measurements.

\section{References}

1. Aiello LP, Gardner TW, King GL, Blankenship G, Cavallerano JD, Ferris FL III, et al. Diabetic retinopathy. Diabetes Care 1998; 21: 143-156.

2. Looker HC, Krakoff J, Knowler WC, Bennett PH, Klein R, Hanson RL. Longitudinal studies of incidence and progression of diabetic retinopathy assessed by retinal photography in Pima Indians. Diabetes Care 2003; 26: 320-326.

3. Stratton IM, Kohner EM, Aldington SJ, Turner RC, Holman RR, Manley SE, et al. UKPDS 50: risk factors for incidence and progression of retinopathy in type II diabetes over 6 years from diagnosis. Diabetologia 2001; 44: 156-163.

4. Adler AI, Stratton IM, Neil HA, Yudkin JS, Matthews DR, Cull CA, et al. Association of systolic blood pressure with macrovascular and microvascular complications of type 2 diabetes (UKPDS 36): prospective observational study. BMJ 2000; 321: 412-419.

5. Cignarelli M, De Cicco ML, Damato A, Paternostro A, Pagliarini S, Santoro $S$, et al. High systolic blood pressure increases prevalence and severity of retinopathy in NIDDM patients. Diabetes Care 1992; 15: $1002-1008$

6. Warpeha KM, Chakravarthy U. Molecular genetics of microvascular disease in diabetic retinopathy. Eye 2003; 17: 305-311.

7. Savage S, Estacio RO, Jeffers B, Schrier RW. Urinary albumin excretion as a predictor of diabetic retinopathy, neuropathy, and cardiovascular disease in NIDDM. Diabetes Care 1996; 19: 12431248.

8. Gall MA, Hougaard P, Borch-Johnsen K, Parving HH. Risk factors 
for development of incipient and overt diabetic nephropathy in patients with non-insulin dependent diabetes mellitus: prospective, observational study. BMJ 1997; 314: 783-788.

9. Parving $\mathrm{HH}$, Hommel E, Mathiesen E, Skott P, Edsberg B, Bahnsen $M$, et al. Prevalence of microalbuminuria, arterial hypertension, retinopathy and neuropathy in patients with insulin dependent diabetes. Br Med J (Clin Res Ed) 1988; 296: 156-160.

10. Wirta O, Pasternack A, Mustonen J, Laippala P, Lahde Y. Retinopathy is independently related to microalbuminuria in type 2 diabetes mellitus. Clin Nephrol 1999; 51: 329-334.

11. Cruickshanks KJ, Ritter LL, Klein R, Moss SE. The association of microalbuminuria with diabetic retinopathy. The Wisconsin Epidemiologic Study of Diabetic Retinopathy. Ophthalmology 1993; 100: 862-867.

12. Park JY, Kim HK, Chung YE, Kim SW, Hong SK, Lee KU. Incidence and determinants of microalbuminuria in Koreans with type 2 diabetes. Diabetes Care 1998; 21: 530-534.

13. Voutilainen-Kaunisto RM, Terasvirta ME, Uusitupa MI, Niskanen LK. Occurrence and predictors of retinopathy and visual acuity in type 2 diabetic patients and control subjects. 10-year follow-up from the diagnosis. J Diabetes Complications 2001; 15: 24-33.

14. Diabetic Retinopathy Study Research Group. Report VII: a modification of the Airlie House classification of diabetic retinopathy. Invest Ophthalmol Vis Sci 1981; 21: 210-226.

15. American Academy of Ophthalmology. http://www.aao.org/education/library/recommendations/upload/Diabetic-Retinopathy-DiseaseSeverity-Scale.pdf; 2006.

16. Gross JL, de Azevedo MJ, Silveiro SP, Canani LH, Caramori ML,
Zelmanovitz T. Diabetic nephropathy: diagnosis, prevention, and treatment. Diabetes Care 2005; 28: 164-176.

17. Deckert T, Feldt-Rasmussen B, Borch-Johnsen K, Jensen T, KofoedEnevoldsen A. Albuminuria reflects widespread vascular damage. The Steno hypothesis. Diabetologia 1989; 32: 219-226.

18. Van Leiden HA, Dekker JM, Moll AC, Nijpels G, Heine RJ, Bouter LM, et al. Risk factors for incident retinopathy in a diabetic and nondiabetic population: the Hoorn study. Arch Ophthalmol 2003; 121: 245-251.

19. Stratton IM, Adler AI, Neil HA, Matthews DR, Manley SE, Cull CA, et al. Association of glycaemia with macrovascular and microvascular complications of type 2 diabetes (UKPDS 35): prospective observational study. BMJ 2000; 321: 405-412.

20. United Kingdom Prospective Diabetes Study Group. Overview of 6 years' therapy of type II diabetes: A progressive disease. Diabetes 1995; 44: 1249-1258.

21. United Kingdom Prospective Diabetes Study Group. Intensive bloodglucose control with sulphonylureas or insulin compared with conventional treatment and risk of complications in patients with type 2 diabetes (UKPDS 33). Lancet 1998; 352: 837-853.

22. Costa LA, Canani LH, Lisboa HR, Tres GS, Gross JL. Aggregation of features of the metabolic syndrome is associated with increased prevalence of chronic complications in type 2 diabetes. Diabet Med 2004; 21: 252-255.

23. Klein R, Klein BE, Moss SE. Is obesity related to microvascular and macrovascular complications in diabetes? The Wisconsin Epidemiologic Study of Diabetic Retinopathy. Arch Intern Med 1997; 157: 650-656. 